\title{
Commentary: Functional assessment of circle of Willis-interesting observation or critical consideration?
}

\author{
Christian D. Etz, MD, PhD, and Michael A. Borger, MD, PhD
}

\author{
From the University Clinic of Cardiac Surgery, Heart Center, University of Leipzig, Leipzig, Germany. \\ Disclosures: Authors have nothing to disclose with regard to commercial support. \\ Received for publication Jan 28, 2019; accepted for publication Jan 28, 2019; available ahead of print March 7 \\ 2019. \\ Address for reprints: Michael A. Borger, MD, PhD, Leipzig Heart Center, University of Leipzig, Struempell- \\ strasse 39, 04289 Leipzig, Germany (E-mail: michael.borger@helios-gesundheit.de). \\ J Thorac Cardiovasc Surg 2019;158:1305-6 \\ $0022-5223 / \$ 36.00$ \\ Copyright (C) 2019 Published by Elsevier Inc. on behalf of The American Association for Thoracic Surgery \\ https://doi.org/10.1016/j.jtcvs.2019.01.105
}

Cerebral protection is paramount for the performance of safe aortic arch repair surgery. A recent survey initiated by the European Association for Cardio-Thoracic Surgery Vascular Domain of more than 100 European centers revealed that bilateral antegrade selective cerebral perfusion (biASCP) is performed in most centers, usually with $22^{\circ} \mathrm{C}$ to $26^{\circ} \mathrm{C}$ perfusate temperature and perfusate flow and pressure in the range of 10 - to $15-\mathrm{mL} / \mathrm{kg}$ and $60 \mathrm{~mm} \mathrm{Hg}$, respectively. ${ }^{1}$ In the United States, however, biASCP use is much less common, with markedly higher proportions of unilateral antegrade selective cerebral perfusion (uASCP), retrograde cerebral perfusion, and hypothermic circulatory arrest techniques. ${ }^{2}$

The evidence in support of either UASCP or biASCP is inconclusive. Although some surgeons use biASCP-presumably to deliver a more physiologically appropriate blood supply - others prefer uASCP because of its technical simplicity and the reduced manipulation of supra-aortic vessels, which may result in a lower risk of iatrogenic embolism or injury to the left common carotid artery. ${ }^{3}$ In their study reported in this issue of the Journal, Smith and colleagues ${ }^{4}$ focus on the functionality of the circle of Willis (CoW), which may present a new and promising technique during aortic arch surgery. Smith and colleagues ${ }^{4}$ describe identification of the patient's individual intracranial arterial collateralization with transcranial Doppler ultrasound (TCD) and present a decision-making algorithm for optimal cannulation and perfusion strategy in aortic arch repair.

Collateral arterial pathways and their clinical evaluation are highly complex issues. Before we conclude that cerebral protection strategies should be based on TCD characterization of the CoW, there are several issues to be addressed. First, TCD is not applicable in approximately $20 \%$ of patients because of poor acoustic transcranial windows, ${ }^{4}$ and an additional $15 \%$ of patients in the study of Smith and colleagues ${ }^{4}$ did not tolerate carotid compression for assessment of CoW function. Carotid compression should also be avoided in patients with severe carotid atherosclerosis, found in approximately $3 \%$ of patients undergoing aortic

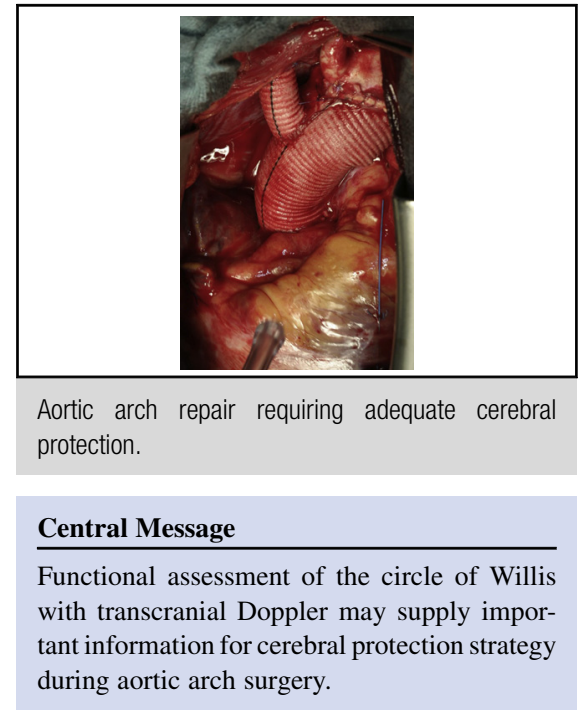

See Article page 1298.

arch repair. The protocol and proposed risk-stratification strategy of Smith and colleagues ${ }^{4}$ is not applicable in 4 out of 10 patients undergoing aortic arch surgery. Second, the clinical relevance of the functional CoW anatomy is debatable, given that excellent clinical outcomes have been reported by centers solely applying uASCP during arch surgery. ${ }^{5-7}$ Local cerebral malperfusion may be attenuated by the use of hypothermia in such series. Indeed, the neurologic complication rates reported in these studies are much lower than would be expected, given the $28 \%$ rate of inadequate unilateral supply identified by the TCD classification system of Smith and colleagues. ${ }^{4}$ Third, hemodynamics at the time of initial TCD evaluation are very different than those observed during the operative repair of the arch. That is, the effects of anesthesia, nonpulsatile blood flow, hypothermia, and lower mean pressures are not taken into account during initial assessment of CoW functionality. Finally, TCD is highly operator dependent and has not found its way into routine use in the cardiac surgery operating room.

Consequently, the proposed TCD assessment of CoW functionality is not applicable in a significant number of patients, and its clinical relevance for the majority of patients remains unclear. In our opinion, however, TCD testing has the potential to become a powerful tool in identifying patients at high risk during arch surgery. More clinical data 
will be required to validate preoperative TCD assessment with intraoperative findings during antegrade selective cerebral perfusion, and such information may lead to adjustments in cerebral perfusion strategy in individual patients (eg, switching from uASCP to biASCP). Whether intraoperative TCD assessment of the CoW is enough to prove or disprove the safety and efficacy of uASCP, an area of controversy for many years, remains to be seen.

In summary, Smith and colleagues ${ }^{4}$ present stimulating data on an innovative approach that may serve as an additional adjunct to improve the safety of arch surgery. Proper interpretation of the data from a technology that has been used sparingly in cardiac surgery will be required, however, and further refinement of the proposed algorithm will almost certainly occur. If TCD functional assessment of the CoW leads to a reduction of cerebral complications (eg, cerebral malperfusion in patients with insufficient intracerebral collateralization, or iatrogenic embolism from unnecessary carotid artery manipulation in patients with robust intracerebral collateralization), then the added effort will be well justified.

\section{References}

1. De Paulis R, Czerny M, Weltert L, Bavaria J, Borger MA, Carrel TP, et al. Current trends in cannulation and neuroprotection during surgery of the aortic arch in Europe. Eur J Cardiothorac Surg. 2015;47:917-23.

2. Englum BR, He X, Gulack BC, Ganapathi AM, Mathew JP, Brennan JM, et al. Hypothermia and cerebral protection strategies in aortic arch surgery: a comparative effectiveness analysis from the STS Adult Cardiac Surgery Database. Eur J Cardiothorac Surg. 2017;52:492-8.

3. Etz CD, Plestis KA, Kari FA, Silovitz D, Bodian CA, Spielvogel D, et al. Axillary cannulation significantly improves survival and neurologic outcome after atherosclerotic aneurysm repair of the aortic root and ascending aorta. Ann Thorac Surg. 2008;86:441-6; discussion 446-7.

4. Smith T, Jafrancesco G, Surace G, Morshuis WJ, Tromp SC, Heijmen RH. A functional assessment of the circle of Willis before aortic arch surgery using transcranial Doppler. J Thorac Cardiovasc Surg. 2019; 158:1298-304.

5. Zierer A, Risteski P, El-Sayed Ahmad A, Moritz A, Diegeler A, Urbanski PP. The impact of unilateral versus bilateral antegrade cerebral perfusion on surgical outcomes after aortic arch replacement: a propensity-matched analysis. J Thorac Cardiovasc Surg. 2014;147:1212-7; discussion 1217-8.

6. Misfeld M, Leontyev S, Borger MA, Gindensperger O, Lehmann S, Legare JF, et al. What is the best strategy for brain protection in patients undergoing aortic arch surgery? A single center experience of 636 patients. Ann Thorac Surg. 2012;93:1502-8.

7. Bachet J. What is the best method for brain protection in surgery of the aortic arch? Selective antegrade cerebral perfusion. Cardiol Clin. 2010;28: $389-401$. 